\title{
The Italian Society of Rheumatology clinical practice guidelines for the management of polymyalgia rheumatica
}

\author{
N. Ughi ${ }^{1,2}$, G.D. Sebastiani ${ }^{3}$, R. Gerli ${ }^{4}$, C. Salvarani ${ }^{5}$, S. Parisi ${ }^{1,6}$, A. Ariani ${ }^{1,7}$, \\ I. Prevete ${ }^{1,3}$, M. Manara ${ }^{1,8}$, F. Rumi', C.A. Scirè ${ }^{1,9}$, A. Bortoluzzi ${ }^{1,9}$ \\ ${ }^{1}$ Epidemiology Unit, Italian Society for Rheumatology, Milan, Italy; ${ }^{2}$ Division of Rheumatology, \\ ASST Grande Ospedale Metropolitano Niguarda, Milano, Italy; ${ }^{3}$ Rheumatology Unit, Azienda Ospedaliera \\ San Camillo-Forlanini, Roma, Italy; ${ }^{4}$ Rheumatology Unit, Department of Medicine, University of Perugia, \\ Perugia, Italy; ${ }^{5}$ Rheumatology Unit, Department of Internal Medicine, Azienda Ospedaliera ASMN IRCCS, \\ Reggio Emilia, Italy; ${ }^{6}$ Rheumatology Unit, Azienda Ospedaliera Città della Salute e della Scienza di Torino, \\ Torino, Italy; ' Department of Medicine, Internal Medicine and Rheumatology Unit, \\ Azienda Ospedaliero-universitaria di Parma, Parma, Italy; ${ }^{8}$ Department of Rheumatology, \\ ASST Gaetano Pini-CTO, Milano, Italy; ${ }^{9}$ Department of Medical Sciences, Rheumatology Section, \\ University of Ferrara, Azienda Ospedaliero-Universitaria Sant'Anna di Cona, Ferrara, Italy
}

\section{SUMMARY}

Objective: to provide evidence-based up-to-date recommendations for the management of patients with a definite diagnosis of polymyalgia rheumatica (PMR).

Methods: A systematic literature review was performed to find the existing clinical practice guidelines (CPGs) on PMR and the framework of the Guidelines International Network Adaptation Working Group was used to appraise (AGREE II), synthesize, and customize the recommendations according to the needs of the Italian healthcare context. Rheumatologists on behalf of the Italian Society of Rheumatology (SIR) and from the SIR Epidemiology Unit joined the working group and identified the key health questions on PMR to guide the systematic literature review. Physicians, including general practitioners and specialists, and health professionals who manage PMR in the clinical practice were the target audience. The final recommendations were rated externally by a multi-disciplinary and multi-professional group of stakeholders.

Results: From the systematic search in databases (Medline, Embase) and grey literature, 3 CPGs were identified and appraised by two independent raters. Combining the statements and the evidence from these CPGs, 9 recommendations were developed by endorsement or adaptation in response to the initial key health questions. The quality of evidence was graded and the working group discussed the final recommendations in view of their implementation in the Italian healthcare context.

Conclusions: In absence of national guidelines so far, these recommendations are the first to provide guidance for the management of patients with a diagnosis of PMR in Italy and they are expected to ensure the best evidence-based clinical practice for this disease.

Keywords: Clinical Practice Guideline, recommendations, polymyalgia rheumatica, management, treatment.

Reumatismo, 2020; 72 (1): 1-15

\section{INTRODUCTION}

olymyalgia rheumatica (PMR) is an inflammatory syndrome characterized by pain and stiffness involving the neck, the shoulders and the hips (1). It is almost exclusively a disease affecting adults over the age of 50, most frequently women. Its highest incidence is reported in individuals over 65 and its incidence increases between 70 and 80 years of age (2). A lower incidence of PMR was observed in the Mediterranean population compared to individuals living in Northern European countries $(3,4)$. In Northern Italy, the mean annual incidence in individuals over 50 years of age was equal to 12.7 cases per 100,000 people between 1980 and 1988 (5) and was slightly higher in Southern Italy with 27.4 cases per 100,000 people between 2014 and 2016 (6). The prevalence of PMR is also higher in the Northern European populations and
Corresponding author: Nicola Ughi

Division of Rheumatology ASST Grande Ospedale Metropolitano Niguarda, Milan, Italy P.zza Ospedale Maggiore, 3 20162 Milano (MI), Italy E-mail: n.ughi@reumatologia.it 
in individuals of Scandinavian descent than in other populations (1). Clinical manifestations include pain and stiffness in the shoulders as well as in the proximal aspects of the arms, neck, pelvic girdle and thighs and are usually bilateral. The disease onset is generally fast, but smouldering and progressive forms may be observed. The clinical expression of stiffness is heterogeneous and often partially overlaps with pain (7). It is closely related to function and lasts typically more than 45 minutes in the involved areas, improves progressively from the early morning over the day and worsens after rest. Constitutional symptoms, such as fatigue, weight loss and fever, are frequent (1). The diagnosis of PMR is suspected, whenever typical symptoms are corroborated by typical laboratory test results, such as elevated erythrocyte sedimentation rate (ESR) and/or C-reactive protein (CRP) (1). Ultrasounds (US) or magnetic resonance imaging (MRI) may be helpful to assess the presence of underlying subdeltoid bursitis, biceps tenosynovitis, synovitis of the glenohumeral joints and/ or hip and/or trochanteric bursitis, which increase diagnostic accuracy (8). For this reason, US findings were included in the last American College of Rheumatology (ACR)/European League Against Rheumatism (EULAR) PMR classification criteria (9). Relevant differential diagnoses include elderly rheumatoid arthritis, calcium pyrophosphate dehydrate deposition disease, infections, malignant tumours, and particularly giant cell arteritis that is associated to PMR in between $10 \%$ and $30 \%$ of cases (10). In this regard, all patients with PMR should be carefully assessed for symptoms and signs of giant cell arteritis, including recent headache, abrupt onset of visual disturbances (especially monocular visual loss), jaw claudication, temporal artery tenderness or decreased temporal artery pulsations (11). Early detection, referral and treatment of PMR are essential, but significant variations can be found in the clinical practice, as this disease is often managed in primary or in secondary care by general practitioners, in-hospital and out-of-hospital rheumatologists, and non- rheumatologists (12). The clinical response to therapy, prevention of and monitoring for side effects are essential components of a comprehensive management program for PMR. Clinical practice guidelines (CPGs) are therefore expected to play a key role in achieving optimal management of patients with PMR.

\section{Need for guidance in Italy}

No recommendations endorsed by the Italian Society of Rheumatology (SIR) have been available, so far. In recent years new international guidelines on PMR were published (13-15). Considering the absence of national guidelines for such a prevalent disease, SIR committed to develop a set of recommendations to provide guidance on treatment and management of patients with a definite diagnosis of PMR for the Italian clinical practice setting.

\section{Objective}

To provide evidence-based up-to-date recommendations for the management of patients with a diagnosis of PMR in Italy.

\section{Target patient population}

Adult patients (age $\geq 18$ years) with PMR whose diagnosis was made by a physician.

\section{Target users}

Physicians (rheumatologists, general practitioners, internists, geriatricians, and physical medicine and rehabilitation physicians) and health professionals who treat patients with PMR in primary care, and hospital and community practice settings. Patients, policy makers and those responsible for commissioning care for patients with PMR in the Italian national health system (NHS).

\section{What is covered}

This guideline addressed the treatment and follow-up of patients with PMR. The concurrent suspicion of elderly-onset rheumatoid arthritis or giant cell arteritis was considered for the purpose of differential diagnosis, yet not in terms of its special management for which readers are invited to refer to pertinent guidelines. 


\section{Areas that are not covered}

Giant cell arteritis with or without PMR, elderly-onset rheumatoid arthritis, and remitting seronegative symmetrical synovitis with pitting oedema (RS3PE) were not addressed in this guideline.

\section{MATERIALS AND METHODS}

\section{Approach to guideline development}

The framework of the Guidelines International Network Adaptation Working Group (http://www.g-i-n.net) following the work of the ADAPTE collaboration $(16,17)$ was used to identify, appraise, synthesize, and customize the existing international guidelines according to the needs of the Italian healthcare context.

\section{Members of the working group}

The members of the working group on behalf of SIR were 7 rheumatologists (N.U., A.B., S.P., A.A., M.M., I.P., C.A.S.) and 1 biostatistician (F.R.) from the SIR Epidemiology Unit, who were responsible for the methodology, and three experts clinicians (G.S., R.G., C.S.), who were involved in the development of the guideline by attending a meeting at the $55^{\text {th }}$ SIR National Meeting (November, $21^{\text {st }}-24^{\text {th }} 2018$ ), e-mail discussions, and a web-meeting (March, 29 ${ }^{\text {th }}$ 2019).

\section{Stakeholder involvement}

A multidisciplinary commission of rheumatologists (18), specialists in internal medicine (1), geriatricians (1), and rehabilitation specialists (1), a general practitioner (1), health professionals (2), and a representative of patients' associations (1) were invited on behalf of SIR to assess and rate the draft of these recommendations. No input from, or cooperation with, any pharmaceutical company nor the industry were involved in the development of these recommendations.

\section{Defining the scope}

The working group identified a set of 9 key health questions which were defined using the PIPOH tool with the five items Population, Intervention, Professionals, Outcomes, and Health settings (Table I). The literature review of the existing recommendations on these health topics was performed accordingly.

\section{Inclusion and exclusion criteria}

Guidelines addressing PMR from international scientific societies were included if published between January $1^{\text {st }} 2009$ and January $8^{\text {th }}$ 2019. Languages other than English or Italian could be considered, if translation could be performed.

Reasons for exclusion: study design (randomized controlled trials (RCT) and uncontrolled trials, observational studies, editorials, commentaries, conference abstracts and narrative/systematic reviews); end-ofsearch date before January $1^{\text {st }} 2009$; poor methodology and reporting; non-original CPGs (i.e. duplication or adaptation of recommendations); not addressing the key health questions.

\section{Search strategy}

The PICO (population, intervention, comparator, outcome) framework of the questions was used to perform the systematic literature search for CPGs in Medline (via PubMed) and Embase databases combining the keywords for PMR and CPGs (search terms not shown, but available on request) in accordance with the proposed filters for review of guidelines $(18,19)$. Moreover, the grey literature was searched by examining the repositories of rheumatology scientific societies and guideline clearinghouses ( $h t t$ ps://www.tripdatabase.com/, https://www. nice.org.uk/), the cross-references, and by using a gate-keeper (Google Scholar). The results were screened by 2 independent reviewers (N.U., A.B.), and disagreements were resolved by consensus. The flow chart of the results is shown in Figure 1.

\section{Appraisal of guideline quality}

Two raters (N.U., A.B.) assessed the quality of the CPGs by using the on-line Appraisal of Guidelines Research and Evaluation (AGREE) II instrument (20). Upon completing the AGREE II assessment, a score and a single-item overall assessment of the guideline were formulated per each 
CPGs as "Recommend" (R), "Recommend with Provisos" ( $\left.\mathrm{R}^{*}\right)$, or "Would Not Recommend". The AGREE II scores were not considered as criteria for exclusion.

\section{Level of evidence and strength of recommendation}

In order to reconcile the different grading systems for evidence across the CPGs, each guideline's grading system was reported rating the level of evidence and strength of recommendation by using the Oxford Lev- els of Evidence (21) (Table II). In case of disagreement, the most updated evidence was considered.

\section{Development of the recommendations}

The characteristics of the included CPGs were described by taking into account the guideline developer, topic, country, language, publication year, end-of-search date, and grading system, and the AGREE II scores were synthesized in tables (not shown). Tables describing the guideline characteristics,

Table I - The key health questions which guided the systematic review of the existing guidelines and the development of the new adapted recommendations.

\begin{tabular}{|c|c|c|}
\hline N. & Health questions* & N. recommendation \\
\hline \multicolumn{3}{|c|}{ Clinical assessment of PMR } \\
\hline 1 & $\begin{array}{l}\text { What is the role of the clinical assessment (e.g. age at disease onset, gender, symptom duration before diagnosis, } \\
\text { quick response to glucocorticoids, presence of peripheral arthritis, giant cell arteritis, RS3PE syndrome, } \\
\text { comorbidities, such as cardiovascular diseases, cerebrovascular diseases, peripheral vasculopathy, chronic } \\
\text { obstructive pulmonary disease, interstitial lung disease, osteoporosis, dyslipidaemia, high blood pressure, } \\
\text { glaucoma, cataract, peptic ulcer, diverticular disease, cognitive disorders, renal failure, latent tuberculosis, } \\
\text { chronic hepatitis B and C, and neoplasm), before starting the treatment? }\end{array}$ & I \\
\hline 2 & $\begin{array}{l}\text { What is the role of laboratory tests (erythrocyte sedimentation rate, C-reactive protein, acute phase reactants, } \\
\text { rheumatoid factor, anti-cyclic citrullinated peptide antibody) before starting the treatment? }\end{array}$ & $\|$ \\
\hline 3 & $\begin{array}{l}\text { What is the role of imaging (conventional X-ray, ultrasonography, FDG-PET/CT), before starting or during } \\
\text { the treatment? }\end{array}$ & III \\
\hline 4 & $\begin{array}{l}\text { What is the role of the criteria of referral to the specialist for shared patients' management by primary } \\
\text { and secondary care in case of atypical features or concurrent giant cell arteritis with or without extracranial } \\
\text { manifestations? }\end{array}$ & IV \\
\hline \multicolumn{3}{|c|}{$\begin{array}{ll} & \text { Treatment of PMR } \\
\end{array}$} \\
\hline 5 & What is the effect of the first-line treatment (non-steroidal anti-inflammatory and glucocorticoids)? & V \\
\hline 6 & $\begin{array}{l}\text { What is the effect of the regimen of the first-line treatment in terms of start time, dose, duration, } \\
\text { discontinuation, and route of administration? }\end{array}$ & $\mathrm{VI}$ \\
\hline 7 & $\begin{array}{l}\text { What is the effect of the type and the start time of treatment following the first-line (i.e. disease-modifying } \\
\text { antirheumatic drugs or biologic agents?) }\end{array}$ & VII \\
\hline 8 & What is the effect of the non-pharmacological interventions? & VIIII \\
\hline \multicolumn{3}{|c|}{$\begin{array}{ll}\text { Follow-up of PMR } \\
\end{array}$} \\
\hline 9 & $\begin{array}{l}\text { What is the role of the follow-up in terms of timing, duration, and definition of disease remission and disease } \\
\text { relapse? }\end{array}$ & IX \\
\hline
\end{tabular}

PMR, polymyalgia rheumatica; FDG-PET/CT, fluorodeoxyglucose positron emission tomography-computed tomography; *Only the intervention is reported for each clinical question which was phrased according to this structure: "In patients with polymyalgia rheumatica $(P)$, which is the role of the intervention (I) performed by rheumatologists, general practitioners, specialists in geriatrics, internal medicine, rehabilitation medicine and other healthcare professionals $(P)$ with regard to the outcome $(O)$ in the primary and secondary care, and/or in case of referral to specialists working in the primary care, hospital and university settings $(H)$ ?". The outcomes considered were those reported in the following list as published in (11): disease remission, disease relapse, duration of glucocorticoid therapy, discontinuation of glucocorticoid therapy, development of giant cell arteritis, glucocorticoid side effects (diabetes mellitus/glucose intolerance, osteoporosis, cardiovascular, disease, dyslipidaemia, impaired wound healing, infections, osteonecrosis, myopathy, cataract, glaucoma, atherosclerosis, hypertension, peptic ulcer, weight gain, moon face, dyspnoea, palpitations, fatigue, skin atrophy, bruising, mood disorders), response to glucocorticoid therapy, cumulative glucocorticoid dose, acute phase reactants, patients assessment of global wellbeing, severity/duration of morning stiffness, lowest possible glucocorticoid dose (prednisone equivalent less than $5 \mathrm{mg} /$ day), functional status (Health Assessment Questionnaire or other measures), quality of life (Short Form-36, EQ5D etc.), mortality, hospitalization (due to disease, its complications, co-morbidity and/or treatment related complications), impact on patients' social environment, fatigue, imaging of shoulder/hip, healthcare resource use (health economics), disease activity score. 


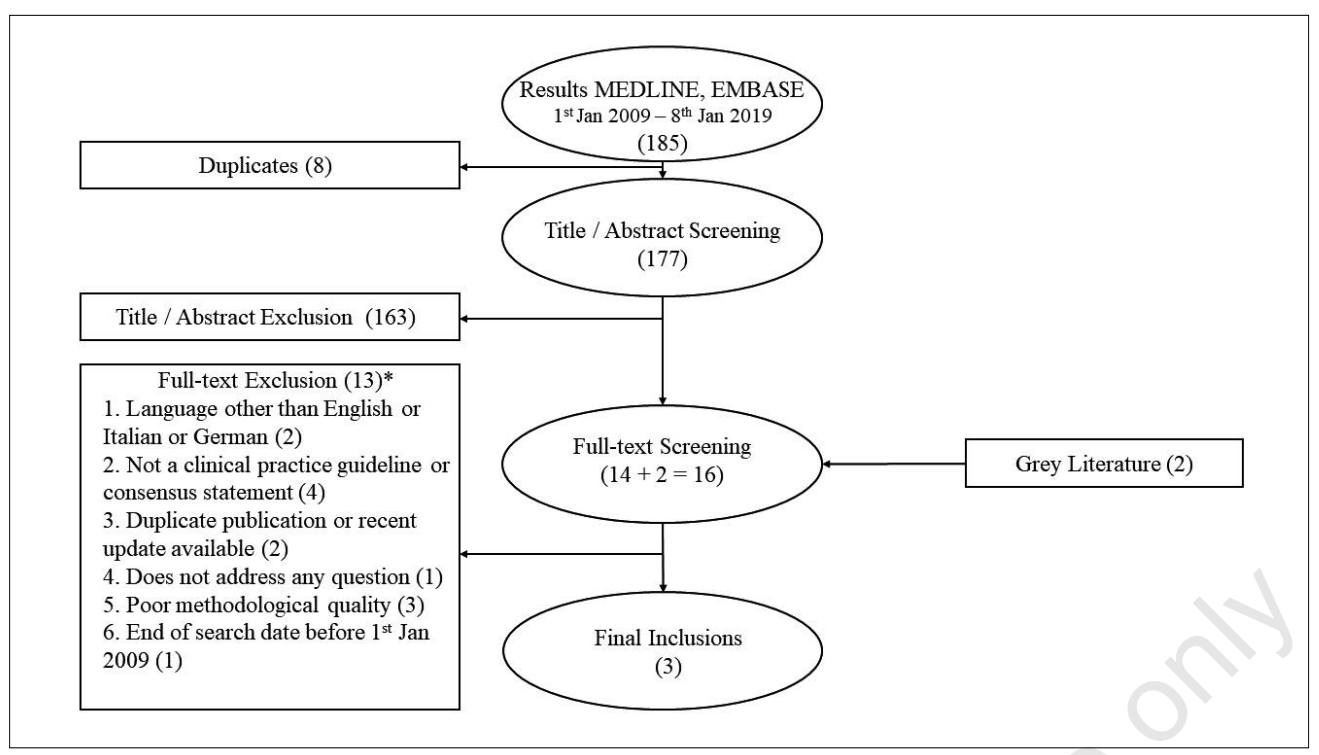

Figure 1 - Steps in the systematic review of guidelines on the management of polymyalgia rheumatica.

*List of the excluded full-texts (reason): Dutch College of General Practitioner's practice guideline on Polymyalgia Rheumatica and temporal arteritis. Eizenga WH, et al. 2010 (1), Diagnostic (classification) criteria and treatment guidelines of collagen-vascular diseases: how to use and cautions on applying them for general physicians. topics: viii. Polymyalgia Rheumatica. Yasuda S, et al. 2015 (1), EULAR/ACR guidelines for the treatment of Polymyalgia Rheumatica. Dasgupta B, et al. 2014 (2), EULAR/ACR guidelines for PMR - the updated evidence. Dejaco C. 2016 (2), 2018 EULAR recommendations for the use of glucocorticoid therapy. Buttgereit F. 2016 (2), Polymyalgia Rheumatica. NICE Clinical Knowledge Summaries. 2013 (2), Concise guidance: diagnosis and management of giant cell arteritis. Dasgupta B, et al. 2010 (3), FDG-PET/ $\mathrm{CT}(\mathrm{A})$ imaging in large vessel vasculitis and polymyalgia rheumatica: joint procedural recommendation of the EANM, SNMMI, and the PET Interest Group (PIG), and endorsed by the ASNC. 2018 (3), EULAR recommendations for the use of imaging in large vessel vasculitis in clinical practice. Dejaco C, et al. 2018 (4), National recommendations based on scientific evidence and opinions of experts on the use of methotrexate in rheumatic disorders, especially in Rheumatoid Arthritis. Results of the $3 E$ Initiative from Brazil. Pereira IA, et al. 2009 (5), Polymyalgia Rheumatica with normal values of both erythrocyte sedimentation rate and C-Reactive Protein concentration at the time of diagnosis: a four-point guidance. Manzo C, et al. 2018 (5), SNLG Regioni. Reumatologia. Linea Guida. Consiglio Sanitario Regionale. Regione Toscana. 2018 (5), BSR and BHPR guidelines for the management of Polymyalgia Rheumatica. Dasgupta B, et al. 2010 (6).

and including the individual recommendations, AGREE summary scores, and the level of evidence and the strength of each recommendation according to the original grading system were built for each key question. Then, each recommendation could be fully adopted or adapted and reworded from the existing recommendation(s). The AGREE reporting checklist was used for reporting this guideline (22).

\section{External review}

A total of 25 stakeholders were consulted to externally review and rate their level of agreement with each draft recommendation (1 to 10-point scoring). An online survey was administered for this purpose via REDcap ${ }^{\circledast}$ between July $16^{\text {th }}$ and Au- gust $1^{\text {st }} 2019$ and the comments from respondents $(n=12$, response rate $48 \%)$ were considered for the development of the final statements of the recommendations. The results of the external review are provided within each recommendation in terms of level of agreement by the stakeholders.

\section{RESULTS}

\section{Key to Understanding This Guidance}

Each recommendation is reported with a level of evidence (LoE), strength of the recommendation (SoR), and level of agreement (LoA) of the stakeholders (Table III). The text of each recommendation is structured as follows: 
Table II - Guidance to categories of evidence and strength of recommendations based on the Oxford Levels of Evidence (21).

\begin{tabular}{|c|l|}
\hline Category & Evidence \\
\hline 1 & From meta-analysis of randomised controlled trials or from at least one randomised controlled trial \\
\hline 2 & From at least one controlled study without randomisation or from at least one cohort study \\
\hline 3 & From at least one case-control study \\
\hline 4 & From case-series or poor-quality cohort and case-control studies \\
\hline 5 & From expert committee reports or opinions and/or clinical experience of respected authorities \\
\hline Grade & Strength \\
\hline A & Consistent level 1 studies \\
\hline B & Consistent level 2 or 3 studies or extrapolations ${ }^{\star}$ from level 1 studies \\
\hline C & Level 4 studies or extrapolations ${ }^{\star}$ from level 2 or 3 studies \\
\hline D & Level 5 evidence or troublingly inconsistent or inconclusive studies of any level \\
\hline
\end{tabular}

*"Extrapolations" are where data is used in a situation that has potentially clinically important differences than the original study situation.

Summary of guidelines. A synthesis of recommendations from the systematic review along with the results of the single-item overall AGREE assessments by the two raters between brackets.

Recommendation/supporting evidence. The original CPG(s) which were used for adaptation.

Evidence to recommendation. Panel's discussion based on the evidence and the clinical experience used to develop the recommendation.

An algorithm which summarizes the pathway for the management of patients with PMR is shown in Figure 2.

\section{RECOMMENDATIONS}

Three original CPGs (13-15) were identified and used to accomplish the final set of 9 recommendations.

\section{Recommendations for the clinical assessment of PMR}

\section{RECOMMENDATION 1: Clinical assessment}

In the presence of sufficient clinical findings to ascertain the PMR case definition, a safe and specific approach should be adopted to exclude relevant

Table III - The final set of 9 recommendations for the management of polymyalgia rheumatica is reported along with the category of evidence, the grading of the recommendation, and the results of the consultation of the stakeholders.

\begin{tabular}{|c|c|c|c|c|}
\hline & The final set of 9 recommendations & LoE & SoR & $\begin{array}{c}\text { LoA, } \\
\text { median (IQR) }\end{array}$ \\
\hline 1 & Clinical assessment & & & \\
\hline & $\begin{array}{l}\text { In the presence of sufficient clinical findings to ascertain the PMR case definition, a safe } \\
\text { and specific approach should be adopted to exclude relevant mimicking or associated conditions, } \\
\text { e.g. non-inflammatory, inflammatory (such as giant cell arteritis or rheumatoid arthritis), } \\
\text { drug-induced, endocrine, infective and neoplastic conditions. } \\
\text { In the management of a patient with PMR the following issue should be considered: } \\
\text { - Determination of comorbidities, particularly hypertension, diabetes, glucose intolerance, } \\
\text { cardiovascular disease, dyslipidaemia, peptic ulcer, osteoporosis (and particularly recent } \\
\text { fractures), presence of cataract or (risk factors for) glaucoma, presence of chronic } \\
\text { or recurrent infections; } \\
\text { - Co-medication with NSAIDs, other relevant medications and risk factors for steroid-related side } \\
\text { effects. } \\
\text { The role of risk factors for relapse/prolonged therapy is not clear yet. Baseline factors that were } \\
\text { associated with a higher relapse rate and/or prolonged therapy in PMR studies were: female sex, } \\
\text { high erythrocyte sedimentation rate (>40 mm/1st hour) and peripheral inflammatory arthritis. }\end{array}$ & 5 & D & $\begin{array}{c}9 \\
(7.75,10)\end{array}$ \\
\hline
\end{tabular}




\begin{tabular}{|c|c|c|c|c|}
\hline & The final set of 9 recommendations & LoE & SoR & $\begin{array}{c}\text { LoA, } \\
\text { median (IQR) }\end{array}$ \\
\hline \multirow[t]{2}{*}{2} & Laboratory assessments & & & \\
\hline & $\begin{array}{l}\text { Every PMR patient should undergo the following assessments prior to the prescription of } \\
\text { therapy and to exclude mimicking conditions and establish a baseline for monitoring of } \\
\text { therapy: C-reactive protein and/or erythrocyte sedimentation rate, rheumatoid factor, anti- } \\
\text { cyclic citrullinated peptide antibodies (ACPA), blood count, glucose, creatinine, liver function } \\
\text { tests, bone profile (including calcium, alkaline phosphatase) and urinalysis. Additional } \\
\text { investigations to consider may be protein electrophoresis, thyroid stimulating hormone (TSH), } \\
\text { creatine kinase. }\end{array}$ & 5 & $\mathrm{D}$ & $\begin{array}{c}9 \\
(8,9.25)\end{array}$ \\
\hline \multirow[t]{2}{*}{3} & Additional investigations & & & \\
\hline & $\begin{array}{l}\text { Additional investigations, such as chest radiography, abdominal ultrasound, and bone } \\
\text { densitometry, may be considered at the discretion of the physician*. } \\
\text { Fluorodeoxyglucose positron-emission tomography combined with computed tomography } \\
\text { (FDG-PET/CT) imaging demonstrated high diagnostic performance in PMRß. However, the } \\
\text { clinical impact of FDG-PET/CT imaging on the management of patients with PMR is still to } \\
\text { be defined and should be considered in a specialist setting. }\end{array}$ & $5^{\star}, 2^{\S}$ & $D^{*}, \mathrm{~B} \S$ & $\begin{array}{c}9 \\
(7.75,9.25)\end{array}$ \\
\hline \multirow[t]{2}{*}{4} & Specialist referral & & & \\
\hline & $\begin{array}{l}\text { Specialist referral should be considered particularly in case of atypical presentation (such } \\
\text { as peripheral inflammatory arthritis, systemic symptoms, low inflammatory markers, age } \\
<60 \text { years), experience of or high risk of therapy-related side effects, PMR refractory to GC } \\
\text { therapy, and/or relapses/prolonged therapy. }\end{array}$ & 5 & $\mathrm{D}$ & $\begin{array}{c}8.5 \\
(7.5,9.25)\end{array}$ \\
\hline \multirow[t]{2}{*}{5} & First-line therapy & & & \\
\hline & $\begin{array}{l}\text { The use of GC instead of NSAIDs in patients with PMR is recommended, with the exception } \\
\text { of possible short-term use of NSAIDs and/or analgesics in PMR patients with pain related to } \\
\text { other conditions. }\end{array}$ & 4 & C & $\begin{array}{c}9 \\
(8,10)\end{array}$ \\
\hline \multirow[t]{2}{*}{6} & Scheduling and route of administration of the first-line therapy & & & \\
\hline & $\begin{array}{l}\text { GC therapy should be started as soon as the diagnosis of PMR is made* } \\
\text { The daily dose of the initial GC therapy should be individualised and the minimum effective } \\
\text { dose within a range of } 12.5-25 \mathrm{mg} \text { prednisone equivalent per day. A higher initial prednisone } \\
\text { dose may be considered in patients with a high risk of relapse and low risk of adverse } \\
\text { events, whereas in patients with relevant comorbidities (e.g., diabetes, osteoporosis, } \\
\text { glaucoma, etc) and other risk factors for GC-related side effects, a lower dose may be } \\
\text { preferred. Initial doses } \leq 7.5 \mathrm{mg} / \text { day should not be used and the use of doses }>30 \mathrm{mg} / \text { day is } \\
\text { not recommended§. } \\
\text { The dose tapering schedules should be individualised and based on regular monitoring of } \\
\text { patient disease activity, laboratory markers and adverse events. The initial tapering should } \\
\text { aim for the dose of } 10 \mathrm{mg} / \text { day prednisone equivalent within } 4-8 \text { weeks. In case of relapse, } \\
\text { the dose should be increased to the pre-relapse dose and decreased gradually (within } 4-8 \\
\text { weeks) to the dose at which the relapse occurred. Once remission is achieved, prednisone } \\
\text { should be tapered by } 1 \mathrm{mg} \text { every } 4 \text { weeks (or by } 1.25 \mathrm{mg} \text { decrements using schedules such } \\
\text { as } 10 / 7.5 \mathrm{mg} \text { alternate days, etc) until discontinuation, if remission is maintained* } \\
\text { Oral GC therapy and the use of a single dose should be preferred to multiple lower doses } \\
\text { across the day for the treatment of PMR*. } \\
\text { The duration of GC therapy in PMR patients should be individualised and be the shortest to } \\
\text { achieve adequate efficacy*. }\end{array}$ & $5^{\star}, 2^{\S}$ & $D^{*}, B^{\S}$ & $\begin{array}{c}9 \\
(9,10)\end{array}$ \\
\hline \multirow[t]{2}{*}{7} & Second-line treatment & & & \\
\hline & $\begin{array}{l}\text { The early introduction of MTX in addition to GCs should be considered particularly in patients at } \\
\text { a high risk of relapse and/or prolonged therapy as well as in cases with risk factors, comorbidities } \\
\text { and/or concomitant medications, where GC-related adverse events are more likely to occur. During } \\
\text { the follow-up, MTX may also be considered in patients with a relapse or experiencing GC-related } \\
\text { adverse events. MTX has been used at oral doses of } 7.5-10 \mathrm{mg} / \text { week in clinical trials. } \\
\text { The use of TNFi for the treatment of PMR is not recommended. } \\
\text { No specific recommendation can be made for other biologic agents, including interelukin-6 } \\
\text { inhibitors. }\end{array}$ & 1 & A & $\begin{array}{c}10 \\
(9.75,10)\end{array}$ \\
\hline
\end{tabular}




\begin{tabular}{|c|c|c|c|c|}
\hline & The final set of 9 recommendations & LoE & SoR & $\begin{array}{c}\text { LoA, } \\
\text { median (IQR) }\end{array}$ \\
\hline \multirow[t]{2}{*}{8} & Non-pharmacological interventions & & & \\
\hline & $\begin{array}{l}\text { An individualised exercise programme should be considered for PMR patients aimed at } \\
\text { maintaining muscular mass and function, and reducing risk of falls, especially in older persons on } \\
\text { long-term GCs as well as in frail patients. }\end{array}$ & 5 & D & $\begin{array}{c}9.5 \\
(8.5,10)\end{array}$ \\
\hline \multirow[t]{2}{*}{9} & Target of the treatment and follow-up & & & \\
\hline & $\begin{array}{l}\text { Treatment of PMR patients should aim at providing the best care and must be based on a } \\
\text { decision shared by the patient and the treating physician. } \\
\text { Patients should have an individualised PMR management plan. Patient perspective and } \\
\text { preferences should be considered in the individualised choice of the initial GC dose and the } \\
\text { subsequent tapering of GCs in PMR. } \\
\text { Patients should have access to education focusing on the impact of PMR and treatment } \\
\text { (including comorbidities and disease predictors) and advice on individually-tailored exercise } \\
\text { programmes. } \\
\text { Every patient treated for PMR in primary or secondary care should be monitored with the } \\
\text { following assessments: risk factors and evidence for steroid-related side effects, comorbidities, } \\
\text { other relevant medications, evidence and risk factors for relapse/prolonged therapy. Follow-up } \\
\text { visits are recommended every 4-8 weeks in the first year, every } 8-12 \text { weeks in the second year, } \\
\text { and as indicated in case of relapse or as prednisone is tapered and discontinued. } \\
\text { It is important for patients to have rapid and direct access to advice from doctors, nurses or } \\
\text { trained healthcare staff to report any changes in their condition, such as flares and adverse } \\
\text { events. }\end{array}$ & & D & $\begin{array}{c}9.5 \\
(8.75,10)\end{array}$ \\
\hline
\end{tabular}

LoE, level of evidence; SoR, strength of the recommendation; LoA, level of agreement of the stakeholders; IQR, interquartile range; PMR, polymyalgia rheumatica; GC, glucocorticoid; NSAID, nonsteroidal anti-inflammatory drug; MTX, methotrexate; TNFi, tumour necrosis factor inhibitors.

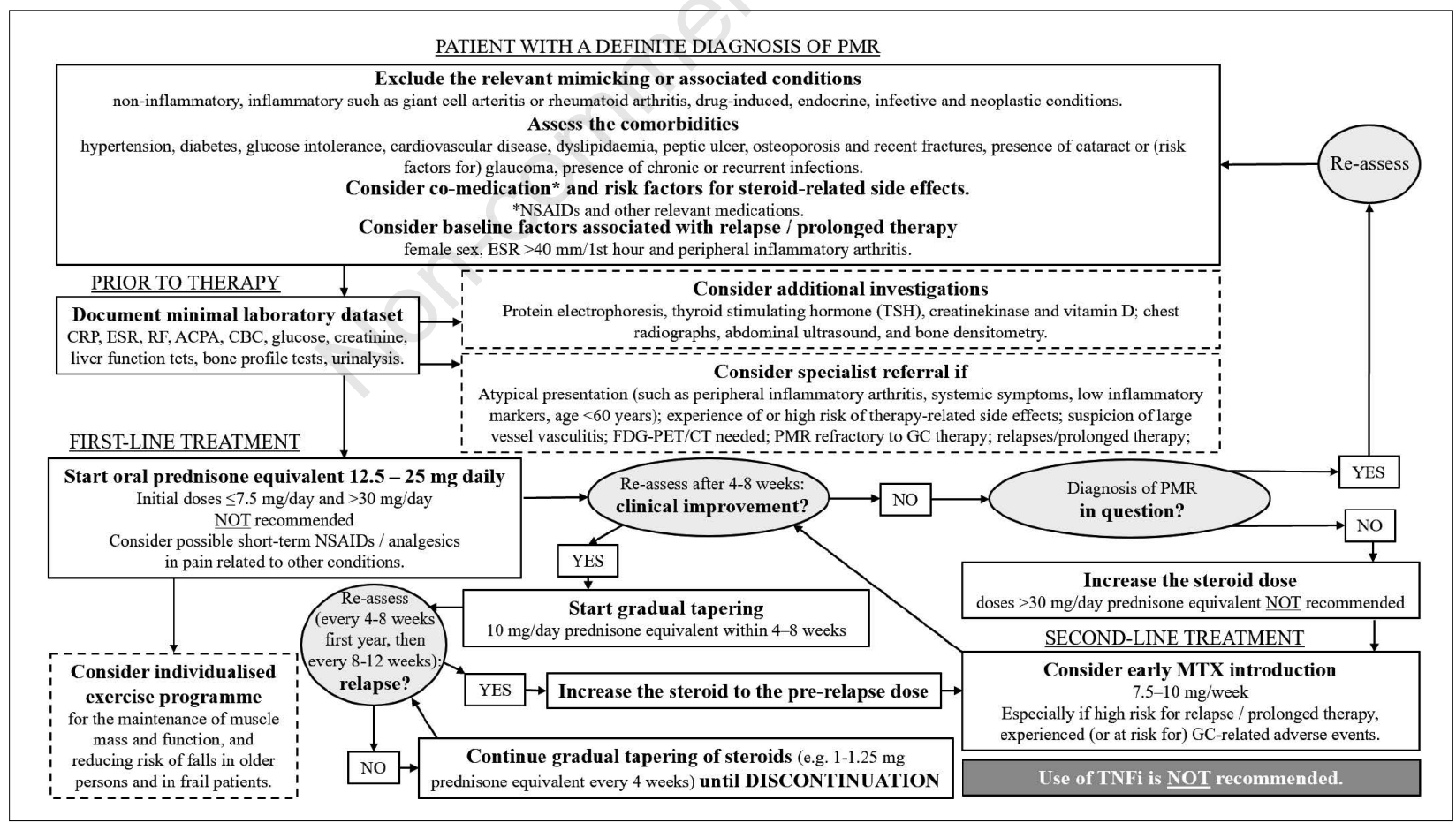

Figure 2 - The management of Polymyalgia Rheumatica according to the recommendations of the Italian Society of Rheumatology. PMR, polymyalgia rheumatica; NSAIDs, nonsteroidal anti-inflammatory drugs; ESR, erythrocyte sedimentation rate; CRP, C-reactive protein; RF, rheumatoid factor; ACPA, anti-cyclic citrullinated peptide antibodies; CBC, cell blood count; GC, glucocorticoids; FDG$\mathrm{PET} / \mathrm{CT}$, fluorodeoxyglucose positron emission tomography-computed tomography; MTX, methotrexate; TNFi, tumour necrosis factor inhibitors. 
mimicking or associated conditions, e.g. non-inflammatory, inflammatory (such as giant cell arteritis or rheumatoid arthritis), drug-induced, endocrine, infective and neoplastic conditions (5, D).

In the management of a patient with PMR the following issues should be considered:

- Determination of comorbidities, particularly hypertension, diabetes, glucose intolerance, cardiovascular disease, dyslipidaemia, peptic ulcer, osteoporosis (and particularly recent fractures), presence of cataract or (risk factors for) glaucoma, presence of chronic or recurrent infections;

- Co-medication with non-steroidal antiinflammatory drugs (NSAIDs), other relevant medications and risk factors for steroid-related side effects $(5, D)$.

The role of risk factors for relapse/prolonged therapy is not clear yet. Baseline factors that were associated with a higher relapse rate and/or prolonged therapy in PMR studies were: female sex, high ESR (>40 mm/1st hour) and peripheral inflammatory arthritis (5, D).

LoE: 5; SoR: D; LoA median (interquartile range): $9.0(7.75,10)$.

Summary of guidelines. The search identified 2 CPGs that addressed the clinical assessment of patients with PMR (AGREE rating: $\mathrm{R}=2$ ).

Recommendation/supporting evidence. ACR/ EULAR 2015 (ACR/EULAR 2015) (13), S3-Leitlinie zur Behandlung der Polymyalgia rheumatica 2018 (S3 2018) (14).

Evidence to recommendation. The panel agreed upon the endorsement of the two CPGs included. The role of CRP was discussed and proposed as an additional baseline factor that could be associated with a higher relapse rate and/or prolonged therapy, yet supported by limited and lowquality evidence $(23,24)$. Moreover, the panel approved the position of the S3 2018 according to which a general or specific tumoral screening is not suggested in addition to the current cancer screening guidelines by age.

\section{RECOMMENDATION 2: Laboratory assessments}

Every PMR patient should undergo the following assessments prior to the prescription of therapy in order to exclude mimicking conditions and establish a baseline for treatment monitoring: CRP and/or ESR, rheumatoid factor, anticyclic citrullinated peptide antibodies (ACPA), blood count, glucose, creatinine, liver function tests, bone profile (including calcium, alkaline phosphatase) and urinalysis. Additional investigations to consider are protein electrophoresis, thyroid stimulating hormone (TSH), creatine kinase (5, D).

LoE 5; SoR D; LoA: 9.0 (8.0, 9.25).

Summary of guidelines. The search identified 2 CPGs that addressed the laboratory assessments of patients with PMR (AGREE rating: $\mathrm{R}=2$ ).

Recommendation/supporting evidence. ACR/EULAR 2015 (13), S3 2018 (14).

Evidence to recommendation. The panel endorsed the recommendation of the two CPGs, but it deemed it appropriate to consider additional more extensive serological tests, such as anti-nuclear antibodies (ANA), anti-cytoplasmic neutrophil antibodies (ANCA) or tuberculosis tests depending on clinical signs and symptoms indicative of other potential diagnoses, and preferably in a specialist setting.

\section{RECOMMENDATION 3: Additional investigations}

Additional investigations, such as chest radiography, abdominal ultrasound, and bone densitometry may be considered at the discretion of the physician $(5, \mathrm{D})$.

Fluorodeoxyglucose positron-emission tomography combined with computed tomography (FDG-PET/CT) imaging demonstrated high diagnostic performance in PMR $(2, B)$. However, the clinical impact of FDG-PET/CT imaging on the management of patients with PMR is still to be defined and should be considered in a specialist setting.

LoE: 2-5; SoR: B-D; LoA: 9.0 (7.75, 9.25). 
Summary of guidelines. The search identified 3 CPGs that addressed the use of additional investigations in patients with PMR (AGREE rating: $\mathrm{R}=3$ ).

Recommendation/supporting evidence. ACR/EULAR 2015 (13), S3 2018 (14), EANM/SNMMI/PIG/ASNC 2018 (15).

Evidence to recommendation. The panel endorsed the recommendations from the CPGs included and added that depending on physician's judgement chest radiography and abdominal ultrasound may be considered to exclude other diagnoses, while bone densitometry may be considered depending on the treatment.

\section{RECOMMENDATION 4:}

\section{Specialist referral}

Specialist referral should be considered particularly in case of atypical presentation (such as peripheral inflammatory arthritis, systemic symptoms, low inflammatory markers, age $<60$ years), experience of or high risk of therapyrelated side effects, PMR refractory to GC therapy, and/or relapses/prolonged therapy.

LoE: 5; SoR: D; LoA: 8.5 (7.5, 9.25).

Summary of guidelines. The search identified 2 CPGs that addressed the referral of patients with PMR to a specialist (AGREE rating: $\mathrm{R}=2$ ).

Recommendation/supporting evidence. ACR/EULAR 2015 (13), S3 2018 (14).

Evidence to recommendation. The panel endorsed the recommendations from the CPGs included.

\section{Recommendations for the treatment of PMR}

\section{RECOMMENDATION 5:}

\section{First-line therapy}

The use of GC instead of NSAIDs in patients with PMR is recommended, with the exception of possible short-term use of NSAIDs and/or analgesics in PMR patients with pain related to other conditions.

LoE: 4; SoR: C; LoA: $8.0(9.0,10)$.
Summary of guidelines. The search identified 2 CPGs that addressed the choice of first-line therapy in patients with PMR (AGREE rating: $\mathrm{R}=2$ ).

Recommendation/supporting evidence. ACR/EULAR 2015 (13), S3 2018 (14).

Evidence to recommendation. The panel endorsed the recommendations from the CPGs included.

\section{RECOMMENDATION 6: Scheduling and route of administration of the first-line therapy}

GC therapy should be started as soon as the diagnosis of PMR is made $(5, D)$.

The daily dose of the initial GC therapy should be individualised and the minimum effective dose within a range of 12.5-25 mg prednisone equivalent per day. A higher initial prednisone dose may be considered in patients with a high risk of relapse and low risk of adverse events, whereas in patients with relevant comorbidities (e.g., diabetes, osteoporosis, glaucoma, etc) and other risk factors for GC-related side effects, a lower dose may be preferred. Initial doses $\leq 7.5 \mathrm{mg}$ /day should not be used and the use of doses $>30 \mathrm{mg} /$ day is not recommended $(2, \mathrm{~B})$.

The dose tapering schedules should be individualised and based on regular monitoring of patient disease activity, laboratory markers and adverse events. The initial tapering should aim for a dose of $10 \mathrm{mg} /$ day prednisone equivalent within 4-8 weeks. In case of relapse, the dose should be increased to the pre-relapse dose and decreased gradually (within 4-8 weeks) to the dose at which the relapse occurred. Once remission is achieved, prednisone should be tapered by $1 \mathrm{mg}$ every 4 weeks (or by $1.25 \mathrm{mg}$ decrements using schedules such as $10 / 7.5 \mathrm{mg}$ on alternate days, etc) until discontinuation, if remission is maintained $(5, \mathrm{D})$.

For the treatment of PMR oral GC therapy and the use of a single dose should be preferred to multiple lower doses across the day $(5, \mathrm{D})$. 
The duration of GC therapy in PMR patients should be individualised and be the shortest to achieve adequate efficacy (5, D).

LoE: 2-5; SoR: B-D; LoA: $9.0(9.0,10)$.

Summary of guidelines. The search identified 2 CPGs that addressed the scheduling and route of administration of the first-line therapy (AGREE rating: $\mathrm{R}=2$ ).

Recommendation/supporting evidence. ACR/EULAR 2015 (13), S3 2018 (14).

Evidence to recommendation. The panel endorsed the recommendations included with regards to the initial dose of GCs, but it did not recommend doses $>25 \mathrm{mg}$ prednisone equivalent per day. From the results of a clinical trial (25), intramuscular (i.m.) methylprednisolone (starting dose of 120 mg methylprednisolone i.m. injection every 3 weeks) can be considered as an alternative to oral GCs, but its use is not common in the Italian setting. The position of the panel was not unanimous with regards to the administration of multiple daily doses of oral GCs in special situations, such as prominent night pain, while tapering GCs below the low-dose range (prednisone or equivalent $<5 \mathrm{mg}$ daily). Such regimen may be considered at the discretion of the treating physician, but it is not the usual clinical practice in the Italian setting.

\section{RECOMMENDATION 7:}

\section{Second-line treatment}

The early introduction of methotrexate (MTX) in addition to GCs should be considered particularly in patients at high risk of relapse and/or prolonged therapy as well as in cases with risk factors, comorbidities and/or concomitant medications, where GC-related adverse events are more likely to occur. During the follow-up, MTX may also be considered in patients with relapse or experiencing GC-related adverse events. MTX has been used at oral doses of 7.5-10 $\mathrm{mg} /$ week in clinical trials $(1, \mathrm{~A})$.

The use of TNF $\alpha$ blocking agents for the treatment of PMR is not recommended $(1, \mathrm{~A})$.
No specific recommendation can be made for other biologic agents, including interleukin-6 inhibitors.

LoE: 1; SoR A; LoA: 10 (9.75, 10).

Summary of guidelines. The search identified 2 CPGs that addressed the choice of second-line therapy in PMR (AGREE rating: $\mathrm{R}=2$ ).

Recommendation/supporting evidence. ACR/EULAR 2015 (13), S3 2018 (14).

Evidence to recommendation. The panel endorsed the recommendations from the CPGs included and agreed that no specific recommendation can be made also for other non-biologic DMARDs due to the absence of clinical studies in PMR, with the exception of hydroxychloroquine, which was ineffective in preventing disease flares in a retrospective clinical study (24).

\section{RECOMMENDATION 8: \\ Non-pharmacological interventions}

An individualised exercise programme should be considered for PMR patients aimed at maintaining muscular mass and function, and reducing risk of falls especially in older persons on long-term GCs as well as in frail patients.

LoE: 5; SoR: D; LoA: 9.5 (8.5, 10).

Summary of guidelines. The search identified 2 CPGs that addressed the nonpharmacological interventions in PMR (AGREE rating: $\mathrm{R}=2$ ).

Recommendation/supporting evidence. ACR/EULAR 2015 (13), S3 2018 (14).

Evidence to recommendation. The panel endorsed the recommendations from the CPGs included.

\section{Recommendations for the follow-up of PMR}

\section{RECOMMENDATION 9:}

Target of treatment and follow-up

Treatment of PMR patients should aim at providing the best care and must be based on a decision shared by the patient and the treating physician $(5, D)$.

Patients should have an individualised PMR management plan. Patient per- 
spective and preferences should be considered in the individualised choice of the initial GC dose and the subsequent tapering of GCs in PMR $(5, \mathrm{D})$.

Patients should have access to education focusing on the impact of PMR and treatment (including comorbidities and disease predictors) and advice on individually-tailored exercise programmes (5, D).

Every patient treated for PMR in primary or secondary care should be monitored with the following assessments: risk factors and evidence for steroidrelated side effects, comorbidities, other relevant medications, evidence and risk factors for relapse/prolonged therapy. Follow-up visits are recommended every 4-8 weeks in the first year, every 8-12 weeks in the second year, and as indicated in case of relapse or as prednisone is tapered and discontinued (5, D).

It is important for patients to have rapid and direct access to advice from doctors, nurses or trained healthcare staff to report any changes in their condition, such as flares and adverse events $(5, \mathrm{D})$. LoE: 5; SoR: D; LoA: $9.5(8.75,10)$.

Summary of guidelines. The search identified 2 CPGs that addressed the target of treatment and follow-up in PMR (AGREE rating: $\mathrm{R}=2$ ).

Recommendation/supporting evidence. ACR/EULAR 2015 (13), S3 2018 (14).

Evidence to recommendation. The panel endorsed the recommendations from the CPGs included.

\section{DISCUSSION AND CONCLUSIONS}

This is the first guidance on management of patients with diagnosis of PMR for clinical practice in Italy, so far. These recommendations were developed from current international consensus and adapted for the context of the NHS.

Glucocorticoids are acknowledged as the mainstay of the first-line PMR treatment. In clinical practice, the initial dose was observed to be between 12.5 and $25 \mathrm{mg}$ prednisone equivalent daily (26), although the scheduling, as well as the duration of therapy, were scarcely investigated (27) and the evidence backing this recommendation is currently limited. Patient reported outcomes, such as visual analogue scale for recording pain measures and fatigue, modified Health Assessment Questionnaire and Medical Outcomes Study Short Form-36 in addition to inflammatory markers, proved to perform well (28) and may help clinicians in tailoring the treatment schedule. The rapid control of symptoms and the regular recovery achieved thanks to the treatment allow the patients to be managed usually in primary care, unless the presence of atypical presentation, unresponsiveness to therapy, relapses and/or prolonged ( $>24$ months) treatment, which is frequently observed (29, 30), may require a specialist referral. In this subset of patients, after a careful re-assessment of the diagnosis to exclude mimicking conditions, like paraneoplastic syndromes, the (early) introduction of MTX is strongly suggested to achieve disease remission as was consistently observed in an Italian cohort of PMR (31). The role of the biological therapy in PMR is still unclear. The use of TNF inhibitors is not recommended on the basis of RCTs with no evidence of large effect due to the administration of infliximab (32) and etanercept (33). The first results from clinical trials on tocilizumab seemed to be promising $(34,35)$, yet still insufficient to provide an evidence basis for clinical guidance. The role of non-pharmacologic treatments were considered as part of a comprehensive approach to PMR, but this recommendation was supported only by expert opinion, since clinical studies are not yet available.

Comorbidities are frequently observed in patients with PMR and may influence the choice of treatment as well as the disease course (36). The development of cataracts may be observed due protracted therapy, but the rates of other morbidities linked to GCs, such as diabetes mellitus, hypertension (requiring medical therapy), and symptomatic vertebral fractures, are not more common in PMR compared to controls of similar age and sex (37). The need 
for a thorough clinical assessment is recommended, because of the widespread clinical experience with a number of conditions which may mimic PMR symptoms, including rheumatoid arthritis, infective, and neoplastic diseases. However, the actual need for additional investigations should be always determined by the treating physician in order to avoid extensive screenings with negligible benefit for the patient compared to the use of the required resources. Particularly, FDG-PET/CT imaging should be used only in the specialist setting, since the indications are mainly limited to confirm the suspicion of concurrent large vessel vasculitis $(8,38)$. However, the role of imaging is still unclear with regards to its probability of detecting large vessel vasculitis in patients with PMR without apparent clinical signs and its potential role in monitoring the efficacy of the treatment.

Finally, the follow-up of PMR patients is heavily influenced by the definitions of disease remission and relapse due to lack of agreement. The treat-to-target strategy may be a valid concept for PMR (39), although further studies are needed before specific outcome measurement sets could be recommended (40) in accordance with patients' priorities (41).

The key strength of these guidelines is the integration of the most recent high-quality international recommendations, while the process of adaptation to the NHS context is ensured by following an acknowledged method. However, there are some limitations. Firstly, the last update of literature search is dependent upon the end-of-search date of the most recent CPGs (July 2016) (14) and subsequent evidence was not considered. Secondly, the majority of recommendations are based on low quality of evidence or expert opinion, particularly with regards to the clinical and laboratory assessment, the GC schedule, nonpharmacological interventions, and disease follow-up. This weakness is due to the absence of (high-quality) studies on PMR and should prompt more research for future recommendations.

In conclusion, these recommendations provide updated guidance including the cur- rent international consensus for the management of PMR for the Italian healthcare context. These are also endorsed by SIR as "guides" only and they do not substitute the individual clinicians' judgment, since they may not apply to all patients and all clinical situations.

\section{Plans for updates}

SIR is committed to review and update these recommendations in the future in order to keep them up-to-date and reflect the development of future treatments or advances in the management of PMR.

\section{Acknowledgements}

The Authors acknowledge all the external reviewers (in alphabetical order those who gave their consent for publication): Bartoletti P., ASL Roma 2, Roma; Borghi A., Università di Bologna, Bologna; Castagnetti M., AUSL Reggio Emilia, Associazione FOR-RHeUMA; Caporali R., Università degli Studi di Milano, Milano; Cimmino MA., Università di Genova, Genova; Dejaco, C., Azienda Sanitaria dell'Alto Adige, Brunico; Delle Sedie A., Azienda Ospedaliero-universitaria di Parma, Parma; Falsetti P., Arezzo; Gerardi MC., Spedali Civili, Università di Brescia, Brescia; Iagnocco A., Università degli Studi di Torino, Torino; Meani L., ASST Gaetano Pini-CTO, Milano; Scrivo R., Sapienza Università di Roma, Roma. We are grateful to Solazzo T. as part of SIR secretariat for administrative support, and to Antonella A. for the translation of the German guidelines into Italian.

\section{Disclosure statements}

N.U. reported a grant from SIR during the conduct of the study and honoraria for consultations from Grünenthal and Laborest Italia outside this submitted work; S.P. reported a grant from SIR and personal fees from Grünenthal, Accord Healthcare, Mylan outside this submitted work; A.A. reported personal fees from Novartis outside this submitted work; M.M. reported a grant from SIR and honoraria for consultations from Ely Lilly, Novartis, Sanofi, MSD, Alfa Wasserman, Celgene outside 
this submitted work; I.P. reported personal fees for advisory board from Abbvie; C.S. reported to be involved in the development of 2015 EULAR/ACR Recommendations for the Management of Polymyalgia Rheumatica; G.D.S., R.G., F.R., C.A.S., and A.B. reported nothing to disclose.

\section{Author Contributions}

All authors were involved in drafting the article or revising critically its important intellectual contents, and all authors approved the final version for publication. Conception and design: N.U., A.B., F.R., S.P., A.A, M.M., I.P., C.A.S. Systematic review search and clinical practice guidelines rating: N.U., A.B. Analysis and discussion of the recommendations: N.U., A.B., G.S., R.G., C.S.

\section{Funding}

No specific funding from public bodies or commercial entities or non-profit organization was granted for drafting these recommendations. Non-economic support, such as meeting rooms and secretarial services, was provided by SIR to carry out the work described in this article.

\section{REFERENCES}

1. González-Gay MA, Matteson EL, Castañeda S. Polymyalgia rheumatica. Lancet $2017 ; 390$ : 1700-12.

2. Gonzalez-Gay MA, Vazquez-Rodriguez TR, Lopez-Diaz MJ, et al. Epidemiology of giant cell arteritis and polymyalgia rheumatica. Arthritis Rheum. 2009; 61: 1454-61.

3. Boesen P, Sørensen SF. Giant cell arteritis, temporal arteritis, and polymyalgia rheumatica in a Danish county. A prospective investigation, 1982-1985. Arthritis Rheum. 1987; 30: 294-9.

4. Schaufelberger C, Bengtsson BA, Andersson R. Epidemiology and mortality in 220 patients with polymyalgia rheumatica. Br J Rheumatol. 1995; 34: 261-4.

5. Salvarani C, Macchioni P, Zizzi F, et al. Epidemiologic and immunogenetic aspects of polymyalgia rheumatica and giant cell arteritis in northern Italy. Arthritis Rheum. 1991; 34: 351-6.

6. De Socio A, Perrotta FM, Grasso GM, Lubrano E. Incidence of rheumatoid arthritis, psoriatic arthritis and polymyalgia rheumatica in an inland area of central Italy: results of the CAMPO-RHE study. Postgrad Med. 2018; 130: 137-41.

7. Mackie SL, Hughes R, Walsh M, et al. "An impediment to living life": why and how should we measure stiffness in polymyalgia rheumatica? PLoS One. 2015; 10 :e0126758.

8. Dejaco C, Ramiro S, Duftner C, et al. EULAR recommendations for the use of imaging in large vessel vasculitis in clinical practice. Ann Rheum Dis. 2018; 77: 636-43.

9. Dasgupta B, Cimmino MA, Kremers HM, et al. 2012 Provisional classification criteria for polymyalgia rheumatica: a European League Against Rheumatism/American College of Rheumatology collaborative initiative. Arthritis Rheum. 2012; 64: 943-54.

10. Michet CJ, Matteson EL. Polymyalgia rheumatica. BMJ 2008; 336: 765-9.

11. Salvarani C, Pipitone N, Versari A, Hunder GG. Clinical features of polymyalgia rheumatica and giant cell arteritis. Nat Rev Rheumatol. 2012; 8: 509-21.

12. Sobrero A, Manzo C, Stimamiglio A. The role of the general practitioner and the out-of-hospital public rheumatologist in the diagnosis and follow-up of patients with polymyalgia rheumatica. Reumatismo 2018; 70: 44-50.

13. Dejaco C, Singh YP, Perel P, et al. 2015 Recommendations for the management of polymyalgia rheumatica: a European League Against Rheumatism/American College of Rheumatology collaborative initiative. Ann Rheum Dis 2015; 74: 1799-807.

14. Buttgereit F, Brabant T, Dinges H, et al. [S3 guidelines on treatment of polymyalgia rheumatica: Evidence-based guidelines of the German Society of Rheumatology (DGRh), the Austrian Society of Rheumatology and Rehabilitation (ÖGR) and the Swiss Society of Rheumatology (SGT) and participating medical scientific specialist societies and other organizations]. Z Rheumatol. 2018; 77: 429-41.

15. Slart RHJA, Members of EANM Cardiovascular, Members of EANM Infection \& Inflammation, Members of Committees, SNMMI Cardiovascular, Members of Council, et al. FDG-PET/CT(A) imaging in large vessel vasculitis and polymyalgia rheumatica: joint procedural recommendation of the EANM, SNMMI, and the PET Interest Group (PIG), and endorsed by the ASNC. Eur J Nucl Med Mol Imaging. 2018; 45: 1250-69.

16. Fervers B, Burgers JS, Haugh MC, et al. Adaptation of clinical guidelines: literature review and proposition for a framework and procedure. Int J Qual Health Care 2006; 18: 167-76.

17. Fervers B, Burgers JS, Voellinger R, et al. Guideline adaptation: an approach to enhance efficiency in guideline development and im- 
prove utilisation. BMJ Qual Saf 2011; 20: 228-36.

18. Geng, Y. PubMed Guidelines filter. 2017. Available at: https://mdanderson.libguides. com/ld.php?content_id=34393248.

19. CADTH's Database Search Filters: Guidelines - OVID Medline, Embase, PsycINFO. Available at: https://www.cadth.ca/resources/ finding-evidence/strings-attachedcadths-database-search-filters.

20. Brouwers MC, Kho ME, Browman GP, et al. AGREE II: advancing guideline development, reporting and evaluation in health care. CMAJ 2010; 182: e839-42.

21. Oxford Centre for Evidence-based Medicine. Levels of Evidence. March 2009. Available at: http://www.cebm.net/oxford-centre-evidencebased-medicine-levels-evidence-march-2009/

22. Brouwers MC, Kerkvliet K, Spithoff K. The AGREE Reporting Checklist: a tool to improve reporting of clinical practice guidelines. BMJ 2016; 352: i1152.

23. Cantini F, Salvarani C, Olivieri I, et al. Erythrocyte sedimentation rate and C-reactive protein in the evaluation of disease activity and severity in polymyalgia rheumatica: a prospective follow-up study. Semin Arthritis Rheum. 2000; 30: 17-24.

24. Lee JH, Choi ST, Kim JS, et al. Clinical characteristics and prognostic factors for relapse in patients with polymyalgia rheumatica (PMR). Rheumatol Int. 2013; 33: 1475-80.

25. Dasgupta B, Dolan AL, Panayi GS, Fernandes L. An initially double-blind controlled 96 week trial of depot methylprednisolone against oral prednisolone in the treatment of polymyalgia rheumatica. Br J Rheumatol. 1998; 37: 189-95.

26. Dubost JJ, Pereira B, Fan A, Soubrier M. What is the starting dose of steroid prescribed by rheumatologist in the management of polymyalgia rheumatica? Ann Rheum Dis 2019 [Epub ahead of print].

27. Campbell AM, Martin JR, Erstad BL. Corticosteroid tapering regimens in rheumatic disease: a systematic review. J Clin Rheumatol 2018 [Epub ahead of print].

28. Matteson EL, Maradit-Kremers H, Cimmino $\mathrm{MA}, \mathrm{S}$ et al. Patient-reported outcomes in polymyalgia rheumatica. J Rheumatol. 2012; 39: 795-803.

29. Giollo A, Rossini M, Bettili F, et al. Permanent discontinuation of glucocorticoids in polymyalgia rheumatica is uncommon but may be enhanced by amino bisphosphonates. J Rheumatol. 2019; 46: 318-22.

30. Albrecht K, Huscher D, Buttgereit F, et al. Long-term glucocorticoid treatment in patients with polymyalgia rheumatica, giant cell arteritis, or both diseases: results from a national rheumatology database. Rheumatol Int. 2018; 38: 569-77.

31. Quartuccio L, Gregoraci G, Isola M, De Vita S. Retrospective analysis of the usefulness of a protocol with high-dose methotrexate in polymyalgia rheumatica: results of a single-center cohort of 100 patients. Geriatr Gerontol Int. 2018; 18: 1410-4.

32. Salvarani C, Macchioni P, Manzini C, et al. Infliximab plus prednisone or placebo plus prednisone for the initial treatment of polymyalgia rheumatica: a randomized trial. Ann Intern Med. 2007; 146: 631-9.

33. Kreiner F, Galbo H. Effect of etanercept in polymyalgia rheumatica: a randomized controlled trial. Arthritis Res Ther. 2010; 12: R176.

34. Lally L, Forbess L, Hatzis C, Spiera R. Brief Report: A prospective open-label phase IIa trial of tocilizumab in the treatment of polymyalgia rheumatica. Arthritis Rheumatol. 2016; 68: 2550-4.

35. Devauchelle-Pensec V, Berthelot JM, Cornec $\mathrm{D}$, et al. Efficacy of first-line tocilizumab therapy in early polymyalgia rheumatica: a prospective longitudinal study. Ann Rheum Dis. 2016; 75: 1506-10.

36. Partington R, Helliwell T, Muller S, et al. Comorbidities in polymyalgia rheumatica: a systematic review. Arthritis Res Ther. 2018; 20: 258.

37. Shbeeb I, Challah D, Raheel S, et al. Comparable rates of glucocorticoid-associated adverse events in patients with polymyalgia rheumatica and comorbidities in the general population. Arthritis Care Res (Hoboken). 2018; 70: 643-7.

38. Prieto-Peña D, Martínez-Rodríguez I, Loricera J, et al. Predictors of positive 18F-FDG PET/CT-scan for large vessel vasculitis in patients with persistent polymyalgia rheumatica. Semin Arthritis Rheum. 2019; 48: 720-7.

39. Camellino D, Dejaco C, Buttgereit F, Matteson EL. Treat to Target: a valid concept for management of polymyalgia rheumatica and giant cell arteritis? Rheum Dis Clin North Am. 2019; 45: 549-67.

40. Owen CE, Yates M, Twohig H, et al. Toward a core outcome measurement set for polymyalgia rheumatica: report from the omeract 2018 special interest group. J Rheumatol. 2019; 46: 1360-4.

41. Morton C, Muller S, Bucknall M, et al. Examining management and research priorities in patients with polymyalgia rheumatica: a primary care questionnaire survey. Clin Rheumatol. 2019; 38: 1767-72. 